\title{
Does Dietary Intake Impact Omentin Gene Expression and Plasma Concentration? A Systematic Review
}

\author{
Mohammad Nosrati-Oskouie ${ }^{a, b}$ Golaleh Asghari $^{b}$ Emad Yuzbashian ${ }^{b}$ \\ Nazanin Sadat Aghili-Moghaddam ${ }^{c,}$ e Maryam Zarkesh $^{d}$ Mohammad Safarian $^{e}$ \\ Parvin Mirmiran ${ }^{b}$ \\ a Student Research Committee, Department of Clinical Nutrition and Dietetics, Faculty of Nutrition Sciences and \\ Food Technology, National Nutrition and Food Technology Research Institute, Shahid Beheshti University of \\ Medical Sciences, Tehran, Iran; ${ }^{\mathrm{b}}$ Nutrition and Endocrine Research Center, Research Institute for Endocrine Sciences, \\ Shahid Beheshti University of Medical Sciences, Tehran, Iran; ' Student Research Committee, Mashhad University \\ of Medical Sciences, Mashhad, Iran; ${ }^{d}$ Cellular and Molecular Endocrine Research Center, Research Institute for \\ Endocrine Sciences, Shahid Beheshti University of Medical Sciences, Tehran, Iran; ${ }^{\mathrm{e}}$ Metabolic Syndrome Research \\ Center, Mashhad University of Medical Sciences, Mashhad, Iran
}

\section{Keywords}

Omentin gene expression · Dietary intakes · Hypocaloric diet $\cdot$ Adipose tissue $\cdot$ Adipokines

\begin{abstract}
Background: Omentin is an adipokine with anti-inflammatory and insulin-sensitizing effects that can play a protective role against cardiovascular disease and diabetes. The aim was to systematically review and summarize the existing evidence on the association between overall dietary intake and omentin gene expression and circulation. Summary: A literature search was conducted in PubMed, Scopus, and Web of Science up to September 2019. Of the 1,940 retrieved articles, 20 relevant studies were included, 6 of which were observational, 11 were clinical trials in humans, and 3 were animal studies. Four randomized controlled trials (RCTs) had a high risk of bias (RoB), 1 had "some concerns", and 2 had a low RoB. Among the nonrandomized studies with comparators, 4 had a serious RoB and 2 had a moderate RoB. In the experimental animal studies with a moderate RoB, conflict-
\end{abstract}

ing results for omentin serum concentration were found for high-fat and low-fat diets. A high-fat diet (HFD) was shown to reduce omentin gene expression in one animal study. In the observational studies, omentin serum concentration was reduced by Ramadan fasting and saturated fatty acid (SFA) intake, and an increase in omentin gene expression was observed with monounsaturated fatty acid (MUFA) intake. There was no association of dietary inflammatory index (DII), macronutrient intake, or total calorie intake with omentin plasma concentrations. In the human interventional studies, omentin plasma concentration increased with a long-term low-calorie, low-fat diet (LFD), and no change was seen with a HFD or a short-term low-calorie diet (LCD). Key Messages: It seems that a long-term diet with a lower fat content and a balanced distribution of fatty acids, i.e., a higher MUFA and lower SFA intake, may effectively increase omentin plasma concentration, possibly via improved insulin resistance and reduced inflammation, but more research is needed to confirm or refute this. c) 2021 The Author(s)

Published by S. Karger AG, Basel
(C) 2021 The Author(s)

Published by S. Karger AG, Basel

This article is licensed under the Creative Commons AttributionNonCommercial-NoDerivatives 4.0 International License (CC BYNC-ND) (http://www.karger.com/Services/OpenAccessLicense) Usage and distribution for commercial purposes as well as any distribution of modified material requires written permission.
Parvin Mirmiran or Emad Yuzbashian

Nutrition and Endocrine Research Center, Research Institute for Endocrine Sciences Shahid Beheshti University of Medical Sciences PO Box 19395-4741, Tehran 1981619573 (Iran) mirmiran@endocrine.ac.ir or e-yuzbashian@yahoo.com 


\section{Introduction}

For decades, it was believed that adipose tissue (AT) was a passive reservoir for fatty acids, and was involved in mechanical and heat insulation, and the regulation of thermogenesis. However, today, it is clear that AT is also an active endocrine organ that secretes a large number of peptides and bioactive mediators called adipokines into the circulation, and that these adipokines have autocrine, paracrine, and endocrine functions. In this context, omentin is one of the adipokines mainly produced by AT and is related to obesity and its comorbidities [1-3].

The omentin gene encodes a $34-\mathrm{kDa}$ adipokine that is expressed and secreted mainly from visceral AT, as well as some other organs such as mesothelial, endothelial, and vascular smooth-muscle cells $[4,5]$. In humans, the omentin gene is located on chromosome 1q21.3 and contains 7 introns and 8 exons [4]. A homolog of omentin with a characteristic of omentin/intelectin has been identified, called omentin-2; omentin-2 has $83 \%$ amino acid similarity to that in omentin [6].

Several studies have shown that omentin plays an important role in maintaining metabolism and insulin sensitivity. It also has anti-inflammatory and antiatherosclerotic effects, and can help to reduce the risk of cardiovascular disease and diabetes through AMP- and mitogen-activated protein kinase, Akt, and NF- $\kappa \mathrm{B}[7,8]$. The main form of omentin in human blood is omentin-1. Past reports showed that a low omentin plasma concentration may contribute to the pathogenesis of insulin resistance and type 2 diabetes mellitus (T2DM) [7, 9]. Also, preclinical and clinical studies reported that omentin plasma concentration was inversely associated with various cardiovascular risk factors, such as body mass index (BMI), waist circumference, body fat, insulin concentration, and homeostasis model assessment of insulin resistance [10-12]. However, a cohort study demonstrated that a higher concentration of omentin is associated with a greater risk of experiencing cardiovascular events in subjects with diabetes. This discrepancy may be due to this fact that omentin plasma concentration may increase in a compensatory manner in response to risk factors that increase cardiovascular events in diabetes [13]. Omentin is also inversely correlated with leptin, resistin, and TNF- $\alpha$, and is positively correlated with adiponectin [8]. The results of these past studies, as well as the many resemblances between omentin and adiponectin, suggests that omentin may be a beneficial adipocytokine [14].

A growing number of studies examining omentin have generated contradictory findings. Some reported
Table 1. PICOS and PECO criteria used to define the research question and search the literature

\begin{tabular}{|c|c|}
\hline Criteria & Description \\
\hline Participants & $\begin{array}{l}\text { Animals (mammals) and human adults (all races } \\
\text { and both sexes) }\end{array}$ \\
\hline $\begin{array}{l}\text { Interventions } \\
\text { /Exposure }^{1}\end{array}$ & $\begin{array}{l}\text { Dietary intake; diet; micro- and macronutrients; } \\
\text { dietary pattern; enriched food }\end{array}$ \\
\hline Comparisons & $\begin{array}{l}\text { With or without a control group in animal and } \\
\text { human studies }\end{array}$ \\
\hline Outcomes & Concentration and gene expression of omentin \\
\hline Study design & $\begin{array}{l}\text { Any interventional trials (animal and human), } \\
\text { observational (longitudinal and cross-sectional) } \\
\text { design }\end{array}$ \\
\hline
\end{tabular}

PICOS: participants, interventions, comparisons, outcomes, study design; PECO: participants, exposure, comparisons, outcomes. ${ }^{1}$ Exposure in observational studies.

that omentin plasma concentration increased after a lowcalorie diet (LCD) $[12,15]$, while others revealed that these diets lead to a reduction in omentin plasma concentration $[16,17]$. There was no change in omentin plasma concentration in response to a very low-calorie diet (VLCD) [18], soybean oil [19], or sesame oil [20]. Interestingly, higher omentin plasma concentrations were detected with lower saturated fatty acid (SFA) intake [21]. However, in another study, no association between omentin plasma concentrations and total energy intake and macronutrients was observed [22]. Since omentin is an adipokine that has anti-inflammatory effects and reduces insulin resistance, and can subsequently prevent cardiovascular disease and diabetes, it is important to understand the relationship between omentin and diet. Because of these inconclusive results on the association between dietary factors and omentin plasma concentrations, summarizing what is known about omentin responses to diet will help to identify knowledge gaps and facilitate the design of future intervention studies.

To date, no systematic review has been conducted with a focus on the association of dietary factors with omentin gene expression and plasma concentration. Therefore, we aimed to summarize the available evidence to clarify the effect of micro- and macronutrients and dietary patterns on omentin gene expression and plasma concentration in humans and animals.
Nosrati-Oskouie et al. 


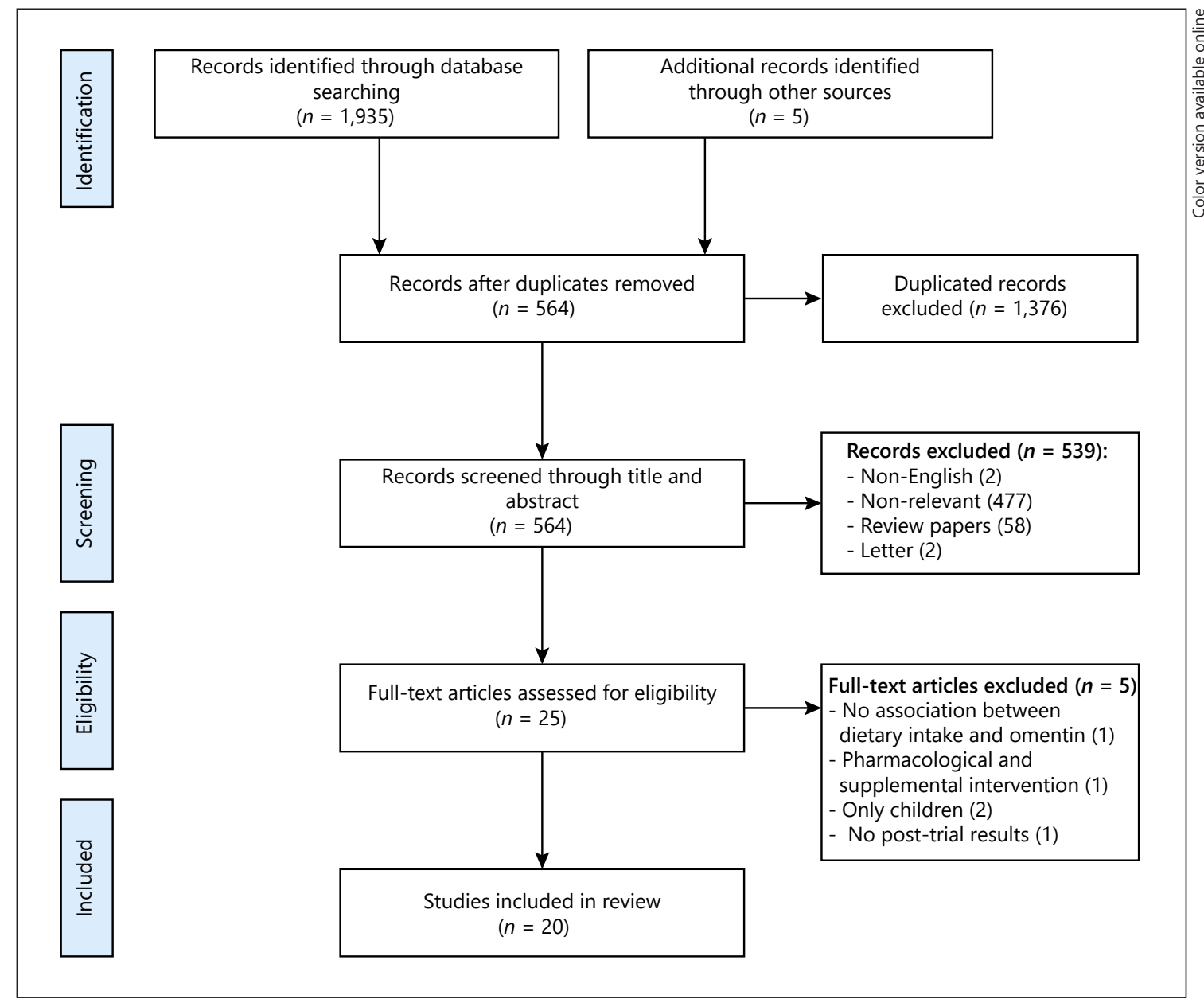

Fig. 1. PRISMA flow diagram for the systematic literature review of the effect of dietary intakes on omentin gene expression and concentration.

\section{Materials and Methods}

\section{Literature Search}

The online databases PubMed, Scopus, and Web of Science were searched for relevant studies. According to the PICOS (participants, interventions, comparisons, outcomes, study design) and PECO (population, exposure, comparison, outcome) inclusion/exclusion criteria for interventional and observational studies, respectively, we used combinations of terms (including $\mathrm{MeSH}$ terms) such as omentin, diet(ary), food, nutrition, nutrient, intake, and pattern to search for relevant publications up to September 2019. Since observational studies were included alongside interventional studies (even without the control group), there was some modification of the PICOS and PECO criteria (Table 1). The reference list of included studies was also checked manually. During the preparation and presentation of this review, the Preferred Reporting Items for Systematic Reviews and Meta-Analysis (PRISMA) guidelines were followed [23]. A summary of the review and the reasons for excluding studies are presented in the PRISMA flow chart (Fig. 1).

\section{Study Eligibility}

Studies were included in this systematic review if they met the following inclusion criteria: (1) they were interventional and observational studies conducted on animals or humans regardless of their demographic group, BMI, age, and sample size; (2) they investigated the association of micro- and macronutrients, food items, food groups, and dietary pattern as exposure, with circulatory concentration and gene expression of omentin; (3) they were published in English, with full texts and not just abstracts. Studies were excluded if they investigated the effect of herbal supplementations and the effect of weight change due to disease without dietary interventions. Case studies, review articles, in vitro studies, and studies on human or animal cell lines were also excluded.

\section{Selecting the Studies}

The screening process commenced with the removal of duplicate articles using EndNote tools. Two researchers independently conducted the literature search and screened the titles, abstracts, and full texts of the identified studies, based on the eligibility criteria detailed above. The final decision regarding the eligibility of articles was made by agreement between the 2 researchers, and any 
disagreement was resolved by involving a third researcher. Figure 1 presents the PRISMA flow diagram of the review summary and procedure.

\section{Data Extraction}

Two investigators independently extracted the following data for each of the included articles: author names, publication year, location, aim, sample size, age, study design, practice setting, study duration, participants' health status, dietary assessment, omentin assessment method, main results, and any other results related to omentin. In the case of missing data or unclear pieces of information, it was considered that the authors did not report such variables.

\section{Assessment of Risk of Bias}

We evaluated the methodological risk of bias (RoB) of the studies using 4 different RoB assessment tools because this review included both human (observational and interventional) and animal studies. The Rob2 tool (revised tool for Risk of Bias in randomized trials) [24] and the ROBINS-I tool (Risk of Bias in Non-Randomized Studies of Interventions) [25] were used for evaluating the RoB of randomized and nonrandomized studies with the comparison between relevant study groups, respectively (online suppl. Fig. 1, 2; see www.karger.com/doi/10.1159/000513885 for all online suppl. material). The RoB of single-arm nonrandomized studies, without evaluating the "comparability" item, was assessed by the Newcastle-Ottawa scale (online suppl. Table 1) [26]. We used the SYRCLE (SYstematic Review Center for Laboratory Animal Experimentation) tool for animal studies (online suppl. Table 2) [27]. Any disagreement was resolved by discussion and consensus. We used the robvis tool (visualization tool for risk of bias assessments in a systematic review) for presenting the data as appropriate [28].

\section{Results}

\section{Overview of Included Studies}

In this analysis, 1,940 articles were identified. A total of 564 articles were screened after removing duplicate articles. After evaluating title and abstract, 25 articles were selected for full-text assessment, 20 of which were identified as meeting the inclusion criteria. Studies were classified according to type, resulting in 6 observational studies [21, 22, 29-32], 3 animal studies [20, 33, 34], and 11 intervention studies. Of the human intervention studies, 7 were conducted without a control group $[12,15-18,35$, $36]$ and 4 considered a control group [19, 37-39].

Participants in the included human studies included both genders and comprised adults between the ages of 18 and 65 years. Of the 17 human studies included in our review, 2 recruited healthy subjects, 10 recruited obese or overweight subjects, and the rest included individuals who were pregnant, diabetic, had nonalcoholic fatty liver disease (NAFLD) or NAFLD with T2DM, or were obese with T1DM. The primary studies were conducted in a variety of countries, including Iran, the Czech Republic, Spain, the USA, and Turkey. A summary of the details and findings of the included studies are presented in Tables 2, 3, and 4, categorized by study design. Because of high methodological heterogeneity (duration, population, and intervention), no meta-analysis was performed.

\section{Animal Experimental Studies}

Up to now, only 3 studies of moderate RoB have assessed the effect of dietary change on the omentin plasma concentration and gene expression in animals (Table 2) [20, 33, 34]. In these studies, a significant reduction in omentin gene expression was observed after intervention with a normal diet and a high-fat diet (HFD) compared to the control (no induced obesity and diabetes) [33, 34]. However, the results regarding the effects of HFD on omentin plasma concentration were contradictory. Feng et al. [33] showed a reduced omentin plasma concentration in the HFD group compared to the control, but no difference was found between the HFD group and the control in another study [34]. Similarly, sesame oil did not show a significant effect on omentin plasma concentration [20]. Also, there were no significant differences in omentin gene expression and plasma concentration between rats with HFD-induced obesity that were fed a LFD and those fed a HFD [33].

In summary, these experimental animal studies, which examined the effects of dietary fat on omentin gene expression, showed that a HFD could reduce omentin gene expression. However, the results regarding omentin plasma concentrations were not consistent with gene expression changes. Consistent and reliable findings of serum and gene expression will be helpful to better understand the function and mechanism involved, and, ultimately, determine the relationships. Therefore, human studies are needed to consider the effect of dietary intake, especially fat, on obesity and diabetes via the regulation of omentin plasma concentration and gene expression.

\section{Human Observational Studies}

Table 3 summarizes the main characteristics and results of the effect of dietary intakes on omentin plasma concentrations. From these studies, we observed that omentin plasma concentrations may be associated with dietary intakes and dietary approaches. The possible effect of Ramadan fasting was demonstrated in 2 moderate RoB studies $[29,30]$. We observed a reduction in omentin serum concentration with fasting compared to nonfasting $[29,30]$; however, Ramadan fasting did not affect omentin serum concentration compared to baseline [30]. One of
Nosrati-Oskouie et al. 


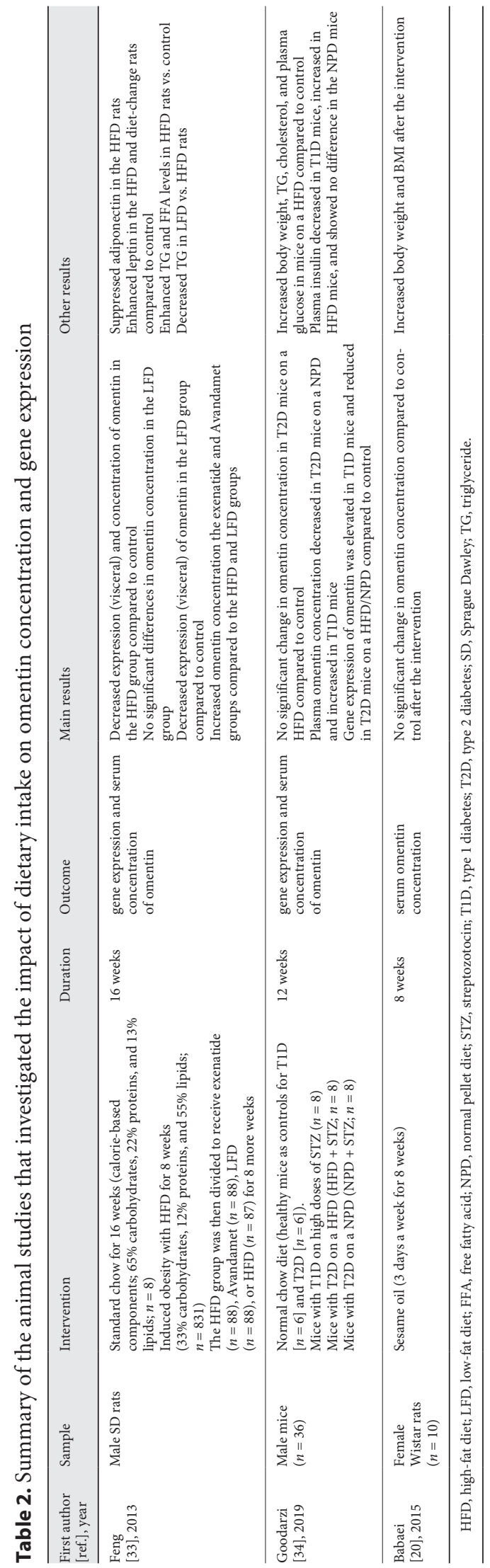

Dietary Intake and Omentin the limitations of this particular study was the lack of a dietary intake assessment [29]. In another high RoB study, we did not find any differences in omentin serum concentration between high and low dietary inflammatory index (DII) scores. It should be mentioned that participants with higher DII scores had a higher intake of carbohydrate and SFAs, and a lower intake of protein, polyunsaturated fatty acids (PUFAs), monounsaturated fatty acids (MUFAs), and fiber [31]. In 2 moderate and high RoB studies, we found no association between omentin plasma concentration and dietary intake of total energy, protein, carbohydrate, and fat [21, 22]. Furthermore, a higher omentin plasma concentration was associated with a lower SFA intake [21]. At the gene expression level, only 1 high RoB study demonstrated a positive association of dietary intake of MUFAs, PUFAs, and omega- 6 with omentin gene expression; however, the observed results remained significant only for dietary MUFAs in the final model [32].

Based on high RoB studies, it seems that SFAs are inversely and MUFAs are positively related to omentin plasma concentration and gene expression, respectively, in participants with obesity. On the other hand, the moderate RoB studies demonstrated that Ramadan fasting could decrease omentin plasma concentration when compared to nonfasting [21, 22, 29-32]. Measurement of dietary intake measurement was variable across studies, with 24-h dietary recall, self-reported record, and food frequency questionnaires all being used. It should be noted that studies have been conducted on participants with obesity and these could indicate the impact of excess body fat accumulation on current findings. However, further observational research including cohort studies with longer follow-up of overweight and normal-weight subjects in larger samples of different ethnicities is needed to substantiate these findings and see if differences exist in different body types. Moreover, the role of confounders, such as insulin, should be considered as a factor that would influence the results of abovementioned studies.

\section{Human Interventional Studies}

Table 4 summarizes the main characteristics and results of dietary intakes on omentin gene expression and plasma concentration in 11 articles. Several studies investigated the effect of a LCD on omentin plasma concentration [12, $15,17,19,35-37]$. In serious RoB studies, we observed that the omentin plasma concentration increased after weight loss from long-term LCDs as well as LCDs within a Mediterranean dietary pattern, in participants with obesity [12, $15,17]$. In studies deemed to be of some concern regarding a RoB, we found similar results with a long-term hypoca- 


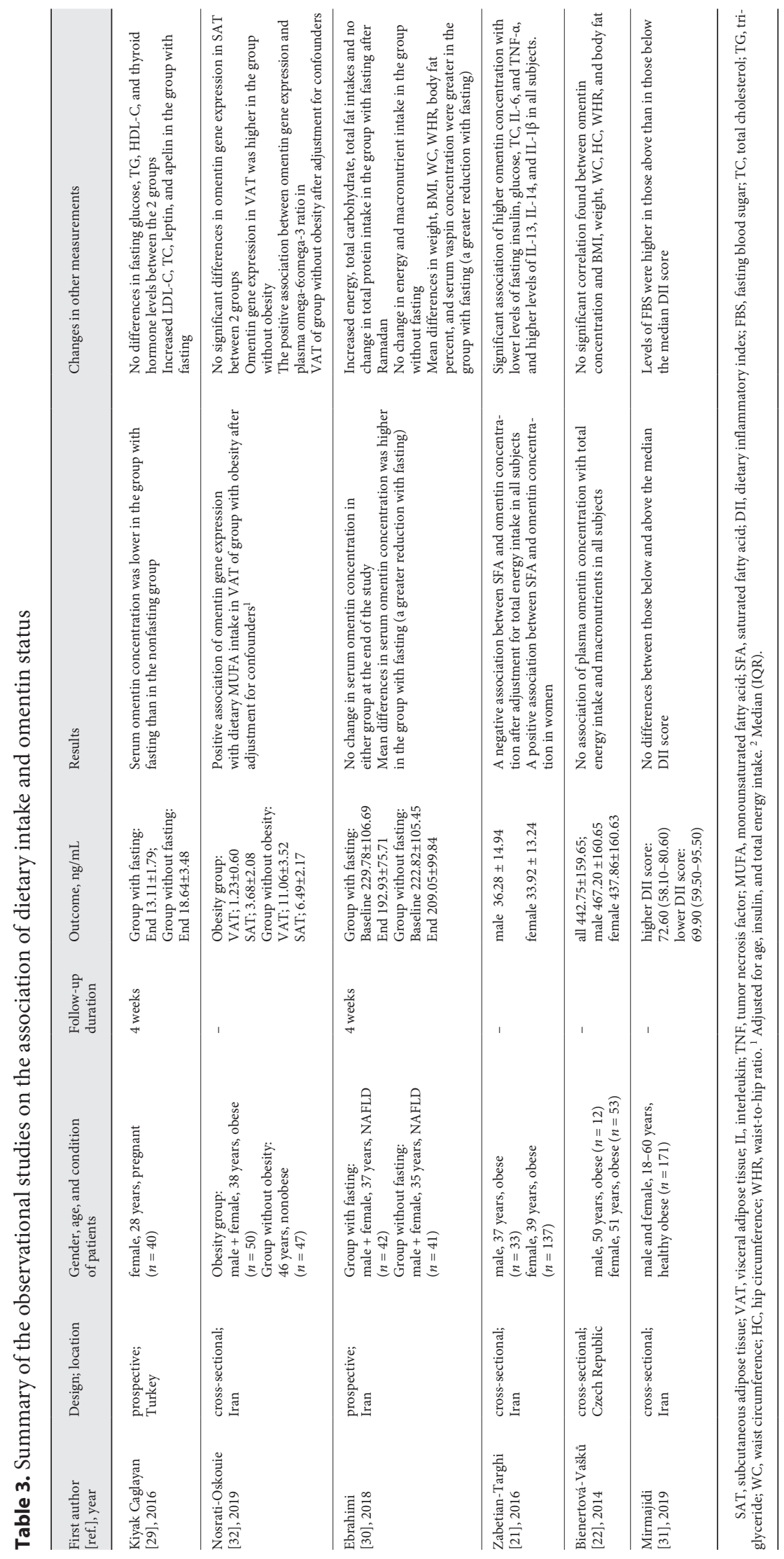




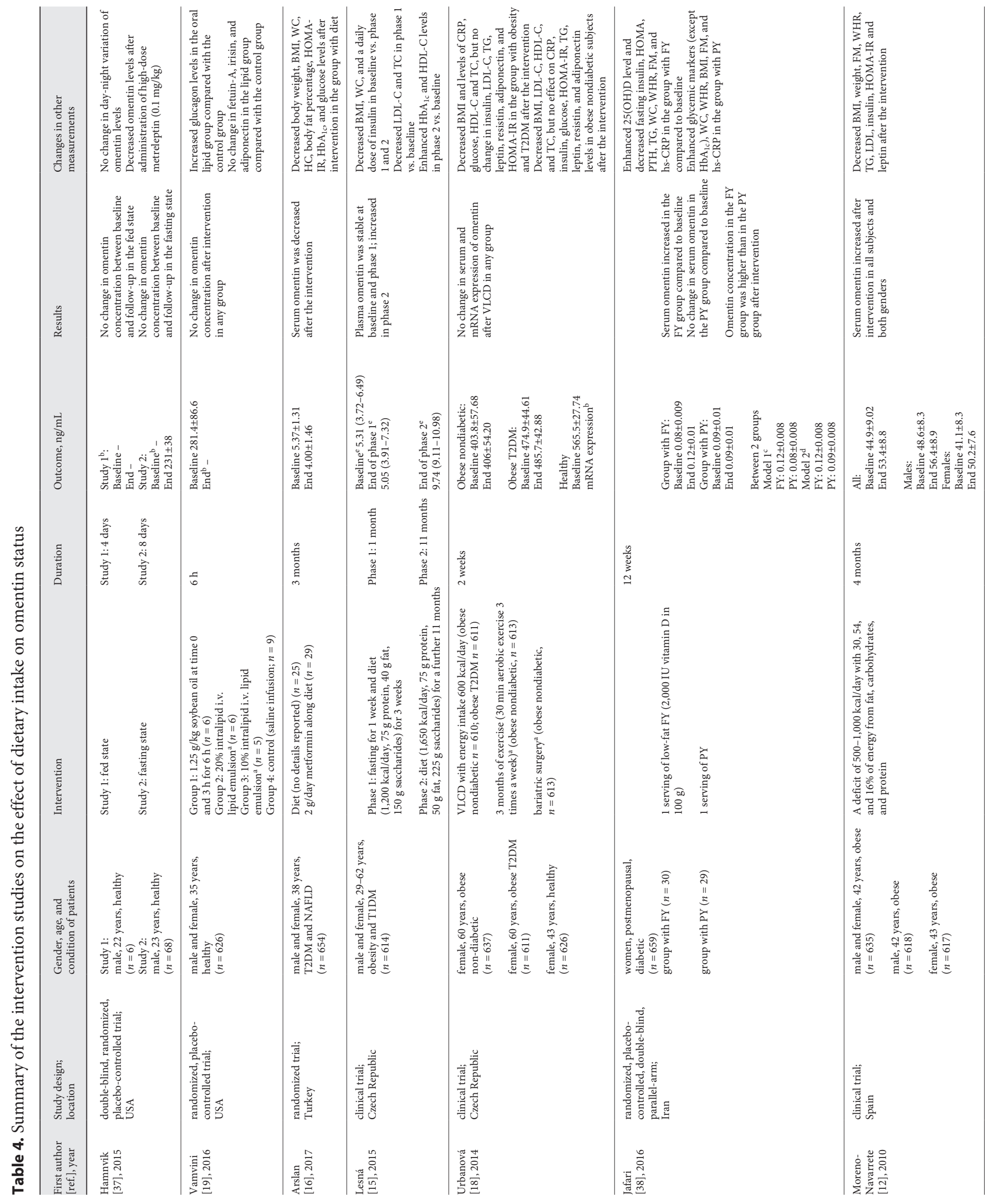




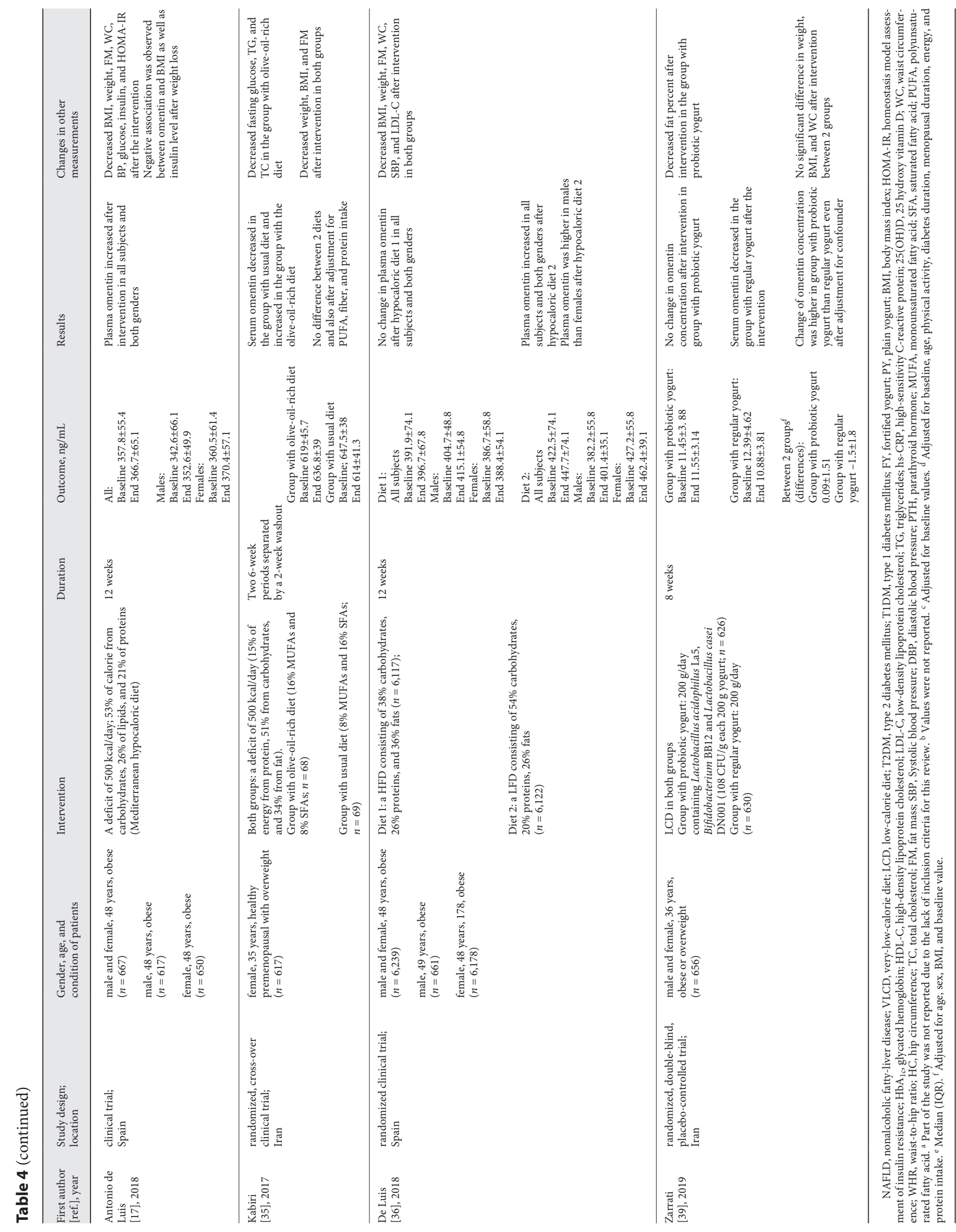




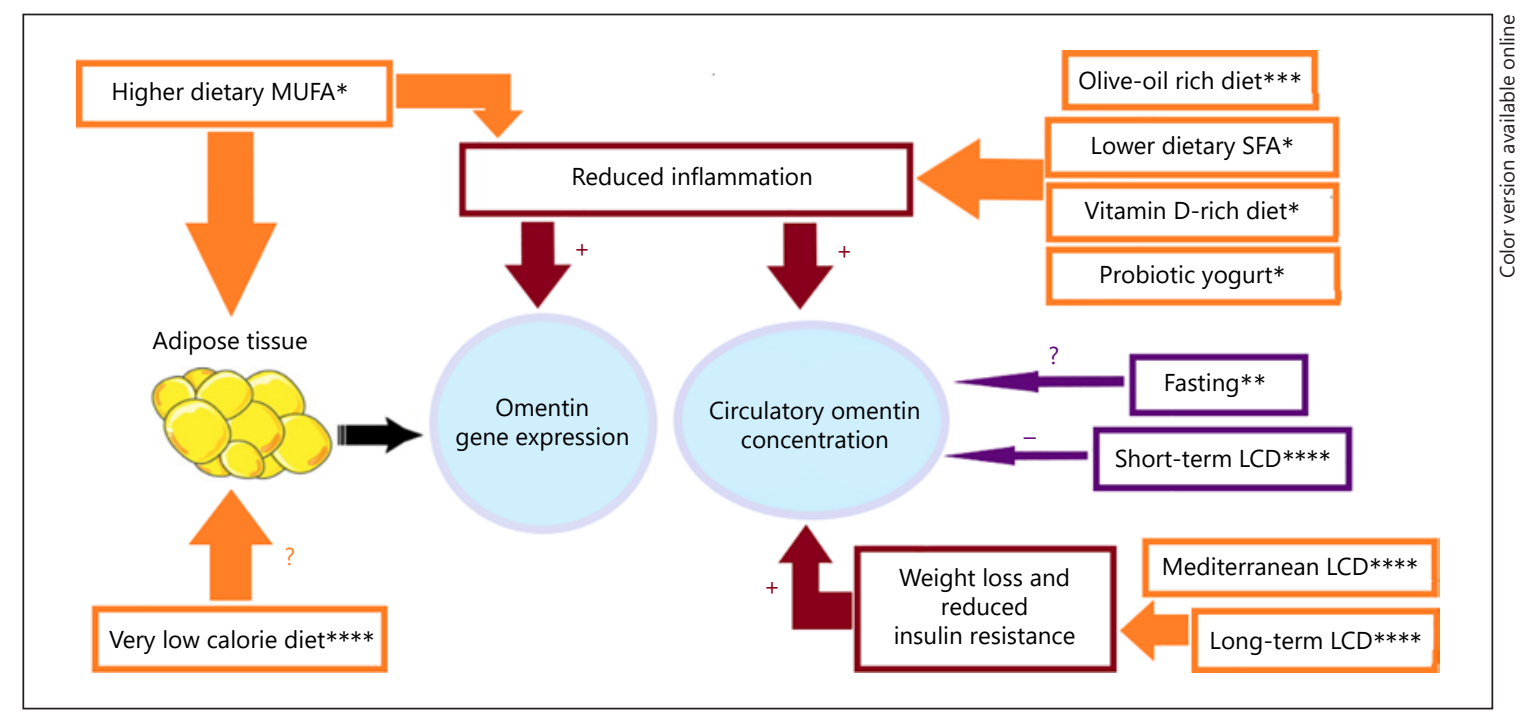

Fig. 2. Schematic representation of dietary impact on omentin status. Red, intermediate mechanism; orange and purple, intervention; +, an increase; -, a decrease; ?, unclear. * a low risk of bias (RoB); ${ }^{* *}$ a moderate RoB; ${ }^{* * *}$ a high RoB; **** a serious RoB.

loric low-fat diet (LFD) [36]. No changes were observed in omentin plasma concentration after intervention with a hypocaloric HFD [36], fasting [37], a short-term LCD [15], or the oral consumption of soybean oil [19]. The distribution of dietary fat was similar in both hypocaloric HFDs and LFDs (45\% MUFAs, 30\% SFAs, and 25\% PUFAs), but omentin plasma concentration was not compared between groups [36]. In this line, only a single study with serious RoB examining the effects of dietary intake on omentin gene expression, in addition to plasma concentration, showed that intervention with a short-term VLCD did not affect omentin plasma concentration and mRNA expression [18]. Also, we did not observe any significant effect of an olive-oil-rich LCD on omentin plasma concentration compared to the control [35].

We found 2 low RoB studies that examined the effects of probiotic yogurt and vitamin $\mathrm{D}$-fortified low-fat yogurt on omentin plasma concentration [38, 39]. These studies showed an increase in omentin plasma concentration and a decrease in high-sensitivity C-reactive protein (hs-CRP) [38]. Similarly, a second study showed that the change in omentin plasma concentration was higher with probiotic yogurt consumption compared to the control [39].

Interventional studies on humans varied with regard to conditions and types of disease, and most of them were focused on dietary energy $[12,15,17,18,35]$ which may subsequently influence weight status and metabolic conditions. Some studies had before-and-after designs without a control group (quasi-experimental) that could affect the validity of the results $[12,15-18,35,36]$. Other studies reported a change in omentin plasma concentration, while it was better to report the effect size. According to the above studies, we found contradictory results regarding the effects of a LCD on omentin. A long-term LCD could increase omentin plasma concentration $[12,15,17]$; however, a short-term LCD had no effect on omentin gene expression and plasma concentration $[15,18]$. Thus, omentin plasma concentration may be potentially affected by dietary fat, the duration of the intervention, and, possibly, the total energy intake, so that omentin increases with a longterm LCD and a LFD. Notably, a long-term LCD that was high in fat, and a short-term LCD rich in MUFAs, did not increase omentin plasma concentration. In general, it can be concluded that short-term dietary interventions do not appear to affect the omentin plasma concentration.

\section{Overall Results}

The abovementioned low RoB observational studies demonstrated that omentin plasma concentrations may have no association with macronutrients except dietary fat, especially SFAs and MUFAs. However, to the best of our knowledge, no interventional studies with this aim have yet been conducted and are needed to confirm this finding by high-quality studies. It seems that the duration of the diet, total caloric intake, and dietary fat, including the amount and type of fat, are among the main determining factors that may impact omentin plasma concentration. 


\section{Discussion}

We systematically reviewed a variety of studies of different designs to address whether dietary intakes affect omentin plasma concentration and/or gene expression. Overall, we found contradictory results regarding the effect of a LCD on the omentin plasma concentrations in humans and animals. In the observational studies, we noted a significant decrease in omentin plasma concentration with fasting and dietary SFA intake, no association with DII, macronutrients, or total calorie consumption, and a positive association between omentin gene expression and dietary intake of MUFAs. The interventional studies reported a significant increase in omentin plasma concentration with the intake of vitamin D-fortified low-fat yogurt, a long-term LCD, and a LFD, but no change with probiotic yogurt, soybean oil, an olive-oil-rich diet, a HFD, a short-term LCD, or a fasting state. In the animal studies, we found that omentin plasma concentration did not change with a HFD (it increased in 1 study), a LFD, and sesame oil intake. Regarding omentin gene expression, a HFD reduced gene expression in animal studies, and a LCD did not affect it in a human study. The results of this review are schematically presented in Figure 2.

The interventional studies showed that a LCD for at least 3 months may be able to increase omentin plasma concentrations in patients with obesity $[12,15,17,36]$. Also, dietary fat appears to have a synergistic effect on the LCD and has a greater impact on omentin plasma concentrations than a LCD. This hypothesis can be supported by studies showing that a LCD with a Mediterranean dietary pattern (of which a high MUFA content is a feature) and a hypocaloric LFD versus a hypocaloric HFD (which did not change omentin plasma concentration despite decreased fat mass), can have a positive effect on increasing the omentin plasma concentration $[17,36]$.

Intervention in the form of a weight reduction program that involved a LCD increased the omentin plasma concentration when insulin resistance improved $[12,17$, $36,40]$. Therefore, calorie reduction does not appear to have an independent effect on the omentin plasma concentration. Weight loss and insulin sensitivity induced by a LCD may affect omentin plasma concentration. We hypothesize that the higher omentin plasma concentration found in participants with obesity and diabetes may be a compensatory mechanism. This hypothesis is supported by the work of Niersmann et al. [13], who reported that omentin was increased in response to cardiometabolic risk factors and inflammation in individuals with a cardiovascular risk.
However, some studies showed a decrease in omentin plasma concentration with a LCD, despite weight loss and improved fasting glucose $[16,18,35]$. We observed that 2 weeks of a VLCD did not affect omentin plasma concentrations and gene expression [18]. It seems that acute energy deprivation may not affect omentin status. Furthermore, Kabiri et al. [35] demonstrated that omentin plasma concentration was decreased after 6 weeks of a LCD with a high SFA content (16\%) compared to a high-MUFA (16\%) diet that increased omentin. Furthermore, in a low RoB study, we found an inverse association between omentin plasma concentration and SFA intake [21]. It seems that the change in omentin plasma concentration might be attributable to dietary fat and fatty acid content. A recent study revealed that the quality and quantity of dietary fatty acids were associated with subcutaneous and visceral $A T$ gene expression [41]. In agreement with this finding, we showed in a previous study that only dietary MUFA intake was related to omentin gene expression [32]. Another possible explanation for these conflicting results found in the literature may be due to the different weight loss interventions, which included a VLCD (acute weight loss) and a LCD with a high SFA content. Finally, a long-term LCD modulated to have reduced fat content (a high MUFA and low SFA intake) is a nutritional approach that may improve the modifiable risk factors of cardiovascular disease, including insulin resistance and omentin plasma concentration; this requires further investigation [35].

Since some studies have demonstrated an inverse correlation between omentin plasma concentrations and inflammatory markers $[42,43]$, we propose that an improved inflammatory status is another possibility for reduced omentin plasma concentrations by modified LCDs and LFDs. We found that a short-term VLCD did not affect omentin plasma concentration and gene expression, or CRP levels, in patients with obesity. Moreover, serum insulin and CRP were higher, and omentin was lower, in patients with obesity and T2DM than in healthy participants [18]. Jafari et al. [38] reported that the intake of vitamin D-fortified low-fat yogurt decreased hs-CRP, and improved inflammation and the omentin plasma concentration, independent of a change in body weight or BMI. Similarly, vitamin D supplementation for 16 weeks decreased hs-CRP while increasing IL-10 and omentin plasma concentration [44]. Omentin acts as an anti-inflammatory factor in patients with diabetic obesity [21], as well as one of the mediators of the antioxidant effect of vitamin D [45].

An observational study showed that fasting for 4 weeks could increase the omentin plasma concentration compared to a nonfasting state [30]. Another study reported
58

Lifestyle Genomics 2021;14:49-61 DOI: $10.1159 / 000513885$
Nosrati-Oskouie et al. 
that omentin plasma concentration in a fasting state for 4 weeks decreased in comparison to not fasting [29]. It should be mentioned that body weight and leptin (that inversely correlated with omentin) increased in pregnant women after a fasting period; this may provide an explanation for the abovementioned discrepancy. Finally, in healthy participants, the clinical trial did not observe any effect of fasting for 8 days on the omentin plasma concentration [37]. It is possible that the lack of an effect of fasting on omentin status was due to the small sample size and short duration of intervention in this particular study. Overall, it seems that fasting for 4 weeks, as is the case in Ramadan fasting, may affect omentin plasma concentration due to weight loss, and it also increases insulin sensitivity.

Omentin plasma concentration may be affected by the amount of dietary fat, the type of dietary fatty acids, weight loss, insulin resistance, inflammation status, and the duration of a dietary intervention. In addition to the quality and quantity of dietary intake, it has been assumed that a change in omentin plasma concentration changes the parameters of metabolic syndrome and insulin sensitivity. Some articles report that the omentin plasma concentration was lower in patients with insulin resistance, T2DM, and obesity than in healthy controls $[5,9,34,46]$. Previous studies suggested that increases in omentin plasma concentration and gene expression might play a protective role against insulin resistance $[12,17,18,21]$. It seems that hyperinsulinemia inhibits omentin secretion $[15,29,36]$. As observed in an animal study, omentin gene expression and plasma concentrations were increased in T1DM mice with low serum insulin [34]. Insulin levels are reduced with weight loss, in association with increased omentin plasma concentrations $[11,12]$. Bearing in mind the results of these studies, increased insulin plasma concentrations, typically found in patients with obesity and T2DM, might indeed be an important contributor that precedes a decrease in omentin plasma concentration. Another possible factor contributing to low omentin plasma concentration could be excessive adiposity and obesity-associated metabolic complications. This possibility is supported by increased omentin plasma concentrations found in patients with anorexia nervosa and severely reduced body fat $[47,48]$.

From the data in the animal study, we observed that omentin gene expression and plasma concentration decreased, and free fatty acids increased, with a HFD [33]. This may be due to the insulin resistance induced by elevated free fatty acids [49]. Also, changing from a HFD to a LFD did not alter the omentin plasma concentration, probably because this intervention did not have any effect on weight loss [33].

Dietary Intake and Omentin
Interestingly, among the relevant studies, Hamnvik et al. [37] failed to demonstrate any change in omentin plasma concentration with chronic energy deprivation and weight loss following bariatric surgery. We observed in another study that, although weight did not change, the plasma concentrations of omentin, glucose, and insulin were different in an aerobic-resistance training group, an estrogen replacement therapy group, and a group that undertook aerobic-resistance training plus estrogen replacement therapy [20]. These results suggest that the omentin plasma concentration is not only affected by weight and fat mass but also by the overall reorganization of fat tissue achieved in the long term. This discrepancy may be explained by the greater loss of subcutaneous versus visceral AT (from which omentin is primarily secreted) following bariatric surgery [50]. Further studies are needed, that focus on omentin expression in different fat deposits, to dissect the potentially distinct roles of subcutaneous AT- and visceral AT-produced omentin.

The major limitation of our systematic review was the limited number of existing interventional studies, especially with longer-term follow-up. Only 4 longitudinal studies had a follow-up period $>3$ months. The follow-up periods of $6 \mathrm{~h}$ to 14 days in the 3 short-term randomized controlled trials (RCTs) were most probably too short to result in any measurable and related effect on omentin plasma concentration. A further limitation was that 4 of the included studies had a cross-sectional design. These studies can suggest associations, but not any causal effects, of diet manipulation on omentin status. The included studies varied widely with regard to study design, population demographics, statistical analyses, and adjustments. Because the association between dietary factors and omentin status was often not the primary research question, the selection of adjustment sets for the control of confounders was not theory-driven. Indeed, most of the analyses did not control for possible omentin-related confounders, which were shown to be linked to dietary factors, such as physical activity. Furthermore, we were unable to perform a meta-analysis, due to the substantial methodological heterogeneity (of durations, populations, and interventions) between included studies. The validity of the assessment tool regarding dietary factors was usually not reported. Many studies were underpowered to detect the interactions of dietary factors and omentin plasma concentrations and gene expression between- or within-groups on account of relatively small sample sizes. The lack of statistical power may explain why the results of the analysis on the effect of dietary factors on omentin status did not reach statistical significance. 


\section{Conclusions}

Based on the current data, there is little compelling evidence that a convincing conclusion can be drawn at present regarding the effect of dietary intake on omentin plasma concentration and gene expression. However, it seems that long-term changes in dietary intake, especially a LFD and a proper distribution of fat content, including a higher MUFA and lower SFA intake, may increase the omentin plasma concentration, possibly via enhanced insulin sensitivity and improved levels of inflammation. However, the evidence remains sparse, mostly due to the lack of highquality studies. Further research is needed to substantiate these findings, given the noted $\mathrm{RoB}$ as well as limitations, inconsistency, and lack of replication of many studies. Longer dietary RCTs which also assess compliance, are necessary to evaluate the efficacy of dietary intake on omentin plasma concentrations and gene expression.

\section{Acknowledgment}

The authors thank Ms. Ghorbani for a critical review of English grammar and syntax.

\section{Statement of Ethics}

This study was approved by the Ethics Committee of the Institute of Endocrinology and Metabolism of Shahid Beheshti University of Medical Sciences (Code of Ethics: IR.SBMU.ENDOCRINE. REC.1398.010).

\section{Conflict of Interest Statement}

The authors declare that they have no competing interests.

\section{Funding Sources}

This study was supported by the Shahid Beheshti University of Medical Sciences, Tehran, Iran (grant No. 22270-7). The funder was not involved in the design of the study, collection, analysis, and interpretation of data, or writing of the manuscript.

\section{Author Contributions}

M.N.-O., E.Y., and N.S.A.-M. designed the research, conducted the literature search and screening, and drafted, reviewed, and revised the manuscript; G.A., P.M., M.S., and M.Z. critically reviewed and revised the manuscript. All authors read and approved the final version.

\section{References}

1 Trayhurn P, Beattie JH. Physiological role of adipose tissue: white adipose tissue as an endocrine and secretory organ. Proc Nutr Soc. 2001 Aug;60(3):329-39.

2 Frühbeck G, Gomez-Ambrosi J. Adipose Tissue: Structure. Function and Metabolism; 2013.

3 Rodríguez A, Ezquerro S, Méndez-Giménez L, Becerril S, Frühbeck G. Revisiting the adipocyte: a model for integration of cytokine signaling in the regulation of energy metabolism. Am J Physiol Endocrinol Metab. 2015 Oct;309(8):E691-714.

4 Schäffler A, Neumeier M, Herfarth H, Fürst A, Schölmerich J, Büchler C. Genomic structure of human omentin, a new adipocytokine expressed in omental adipose tissue. Biochim Biophys Acta. 2005 Dec; 1732(1-3):96-102.

5 Yang RZ, Lee MJ, Hu H, Pray J, Wu HB, Hansen $\mathrm{BC}$, et al. Identification of omentin as a novel depot-specific adipokine in human adipose tissue: possible role in modulating insulin action. Am J Physiol Endocrinol Metab. 2006 Jun;290(6):E1253-61.

6 Lee JK, Schnee J, Pang M, Wolfert M, Baum LG, Moremen KW, et al. Human homologs of the Xenopus oocyte cortical granule lectin XL35. Glycobiology. 2001 Jan;11(1):65-73.
7 Zhou JY, Chan L, Zhou SW. Omentin: linking metabolic syndrome and cardiovascular disease. Curr Vasc Pharmacol. 2014 Jan;12(1): 136-43.

8 Watanabe T, Watanabe-Kominato K, Takahashi Y, Kojima M, Watanabe R. Adipose Tissue-Derived Omentin-1 Function and Regulation. Compr Physiol. 2017 Jun;7(3):765-81.

9 Yoo HJ, Hwang SY, Hong HC, Choi HY, Yang SJ, Seo JA, et al. Association of circulating omentin-1 level with arterial stiffness and carotid plaque in type 2 diabetes. Cardiovasc Diabetol. 2011 Nov; 10(1):103.

10 de Souza Batista CM, Yang RZ, Lee MJ, Glynn NM, Yu DZ, Pray J, et al. Omentin plasma levels and gene expression are decreased in obesity. Diabetes. 2007 Jun;56(6):1655-61.

11 Tan BK, Adya R, Farhatullah S, Lewandowski KC, O'Hare P, Lehnert H, et al. Omentin-1, a novel adipokine, is decreased in overweight insulin-resistant women with polycystic ovary syndrome: ex vivo and in vivo regulation of omentin-1 by insulin and glucose. Diabetes. 2008 Apr;57(4):801-8.

12 Moreno-Navarrete JM, Catalán V, Ortega F, Gómez-Ambrosi J, Ricart W, Frühbeck G, et al. Circulating omentin concentration increases after weight loss. Nutr Metab (Lond). 2010 Apr;7(1):27.
13 Niersmann C, Carstensen-Kirberg M, Maalmi H, Holleczek B, Roden M, Brenner H, et al. Higher circulating omentin is associated with increased risk of primary cardiovascular events in individuals with diabetes. Diabetologia. $2020 \mathrm{Feb} ; 63(2): 410-8$.

14 Yamawaki H. Vascular effects of novel adipocytokines: focus on vascular contractility and inflammatory responses. Biol Pharm Bull. 2011;34(3):307-10

15 Lesná J, Tichá A, Hyšpler R, Musil F, Bláha V, Sobotka L, et al. Omentin-1 plasma levels and cholesterol metabolism in obese patients with diabetes mellitus type 1 : impact of weight reduction. Nutr Diabetes. 2015 Nov;5(11):e18383.

16 Arslan I, Ulas T, Karakas E, Demir M, Eren M, Torun A, et al. Comparative effectiveness of diet alone and diet plus metformin treatment on omentin levels in type 2 diabetes patients with nonalcoholic fatty liver disease: a prospective randomized trial. Period Biol. 2017; 119(1):9-15.

17 Antonio de Luis D, Izaola O, Primo D, Aller R. Modifications of serum levels of omentin-1 and other cardiovascular risk factors following weight loss secondary to a Mediterranean hypocaloric diet. Clin Nutr. 2018 Dec;37(6 Pt A):2280-3. 
18 Urbanová $\mathrm{M}$, Dostálová I, Trachta $\mathrm{P}$, Drápalová J, Kaválková P, Haluzíková D, et al. Serum concentrations and subcutaneous adipose tissue mRNA expression of omentin in morbid obesity and type 2 diabetes mellitus: the effect of very-low-calorie diet, physical activity and laparoscopic sleeve gastrectomy. Physiol Res. 2014;63(2):207-18.

19 Vamvini MT, Hamnvik OP, Sahin-Efe A, Gavrieli A, Dincer F, Farr OM, et al. Differential Effects of Oral and Intravenous Lipid Administration on Key Molecules Related to Energy Homeostasis. J Clin Endocrinol Metab. 2016 May;101(5):1989-97.

20 Babaei P, Pourrahim Ghouroghchi A, Damirchi A, Soltani Tehrani B. The interactive effect of aerobic-resistance training and estrogen therapy on metabolic syndrome indices and omentin-1. Physiol Pharmacol. 2015;19(3): $200-7$.

21 Zabetian-Targhi F, Mirzaei K, Keshavarz SA, Hossein-Nezhad A. Modulatory Role of Omentin-1 in Inflammation: Cytokines and Dietary Intake. J Am Coll Nutr. 2016 NovDec;35(8):670-8.

22 Bienertová-Vašků J, Novák J, Zlámal F, Forejt M, Havlenová S, Jackowská A, et al. The prediction role of indexes of circulating adipokines for common anthropometric and nutritional characteristics of obesity in the obese Central European population. Eat Behav. 2014 Apr;15(2):244-51.

23 Moher D, Liberati A, Tetzlaff J, Altman DG; PRISMA Group. Preferred reporting items for systematic reviews and meta-analyses: the PRISMA statement. PLoS Med. 2009 Jul; 6(7):e1000097.

24 Sterne JA, Savović J, Page MJ, Elbers RG, Blencowe NS, Boutron I, et al. RoB 2: a revised tool for assessing risk of bias in randomised trials. BMJ. 2019 Aug;366:14898.

25 Sterne JA, Hernán MA, Reeves BC, Savović J, Berkman ND, Viswanathan M, et al. ROBINS-I: a tool for assessing risk of bias in nonrandomised studies of interventions. BMI. 2016 Oct;355:i4919.

26 Wells G, Shea B, O'connell D, Peterson J, Welch V, Losos M, et al. The Newcastle-Ottawa Scale (NOS) for assessing the quality if nonrandomized studies in meta-analyses. 2009. Available from: URL: http://www ohri $\mathrm{ca} /$ programs/clinical_epidemiology/oxford htm [cited 2009 Oct 19]. 2016.

27 Hooijmans CR, Rovers MM, de Vries RB, Leenaars M, Ritskes-Hoitinga M, Langendam MW. SYRCLE's risk of bias tool for animal studies. BMC Med Res Methodol. 2014 Mar; 14(1):43.

28 McGuinness LA, Higgins JP. Risk-of-bias VISualization (robvis): an R package and Shiny web app for visualizing risk-of-bias assessments. Res Synth Methods. 2020, Online ahead of print.
29 Kiyak Caglayan E, Engin-Ustun Y, Sari N, Gocmen AY, Polat MF. The effects of prolonged fasting on the levels of adiponectin, leptin, apelin, and omentin in pregnant women. J Obstet Gynaecol. 2016 May;36(4):555-8.

30 Ebrahimi S, Gargari BP, Izadi A, Imani B, Asjodi F. The effects of Ramadan fasting on serum concentrations of vaspin and omentin-1 in patients with nonalcoholic fatty liver disease. Europ J Integr Med. 2018;19:110-14.

31 Mirmajidi S, Izadi A, Saghafi-Asl M, Vahid F, Karamzad N, Amiri P, et al. Inflammatory Potential of Diet: Association with Chemerin, Omentin, Lipopolysaccharide-Binding Protein, and Insulin Resistance in the Apparently Healthy Obese. J Am Coll Nutr. 2019 MayJun;38(4):302-10.

32 Nosrati-Oskouie M, Safarian M, Yuzbashian E, Asghari G, Zarkesh M, Aghili-Moghaddam NS, et al. The association of omentin gene expression in visceral and subcutaneous adipose tissues with plasma fatty acids profile and dietary fatty acids. Majallah-i Ghudad-i Darun/ Riz va Mitabulism-i Iran. 2019;21(3):123-37.

33 Feng WH, Yuan XW, Tong GY, Wang WM, $\mathrm{Hu} \mathrm{Y}$, Shen SM, et al. Correlated increase of omentin-1 and adiponectin by exenatide, avandamet and dietary change in diet-induced obese rats. Folia Biol (Praha). 2013; 59(6):217-24.

34 Goodarzi G, Shirgir A, Alavi S, Khoshi A. Effect of insulin-glucose metabolism compared with obesity on adipose omentin gene expression in different models of diabetic C57BL/6 mice. Diabetol Metab Syndr. 2019 Aug;11:65.

35 Kabiri A, Hosseinzadeh-Attar MJ, Haghighatdoost F, Eshraghian M, Esmaillzadeh A. Impact of olive oil-rich diet on serum omentin and adiponectin levels: a randomized crossover clinical trial among overweight women. Int J Food Sci Nutr. 2017 Aug;68(5):560-8.

36 de Luis DA, Izaola O, Primo D, Aller R. Impact of 2 Different Hypocaloric Diets on Serum Omentin Levels in Obese Subjects. Ann Nutr Metab. 2018;73(2):138-44.

37 Hamnvik OR, Thakkar B, Chamberland J, Aronis K, Schneider B, Mantzoros CS. Omentin-1 levels are reduced by pharmacologic doses of leptin, but remain unaffected by energy deprivation and display no day-night variation. Int J Obes. 2015 Feb;39(2):260-4.

38 Jafari T, Faghihimani E, Feizi A, Iraj B, Javanmard SH, Esmaillzadeh A, et al. Effects of vitamin D-fortified low fat yogurt on glycemic status, anthropometric indexes, inflammation, and bone turnover in diabetic postmenopausal women: A randomised controlled clinical trial. Clin Nutr. 2016 Feb; 35(1):67-76.

39 Zarrati M, Raji Lahiji M, Salehi E, Yazdani B, Razmpoosh E, Shokouhi Shoormasti R, et al. Effects of Probiotic Yogurt on Serum Omentin-1, Adropin, and Nesfatin-1 Concentrations in Overweight and Obese Participants Under Low-Calorie Diet. Probiotics Antimicrob Proteins. 2019 Dec;11(4):1202-9.
40 Zhang M, Tan X, Yin C, Wang L, Tie Y, Xiao Y. Serum levels of omentin-1 are increased after weight loss and are particularly associated with increases in obese children with metabolic syndrome. Acta Paediatr. 2017 Nov; 106(11):1851-6.

41 Rostami H, Samadi M, Yuzbashian E, Zarkesh M, Asghari G, Hedayati M, et al. Habitual dietary intake of fatty acids is associated with leptin gene expression in subcutaneous and visceral adipose tissue of patients without diabetes. Prostaglandins Leukot Essent Fatty Acids. 2017 Nov; 126:49-54.

42 Yu HY, Zhao P, Wu MC, Liu J, Yin W. Serum adropin levels are decreased in patients with acute myocardial infarction. Regul Pept. 2014 May;190-191:46-9.

43 Buyukinan M, Atar M, Can U, Pirgon O, Guzelant A, Deniz I. The Association Between Serum Vaspin and Omentin-1 Levels in Obese Children with Metabolic Syndrome. Metab Syndr Relat Disord. 2018 Mar;16(2): 76-81.

44 Fazelian S, Paknahad Z, Khajehali L, Kheiri S, Amani R. The effects of supplementation with vitamin $\mathrm{D}$ on inflammatory biomarkers, omentin, and vaspin in women with type 2 diabetes: A randomized double-blind placebo-controlled clinical trial. J Food Biochem. 2018;42(6):e12631.

45 Jaganathan R, Ravindran R, Dhanasekaran S. Emerging Role of Adipocytokines in Type 2 Diabetes as Mediators of Insulin Resistance and Cardiovascular Disease. Can J Diabetes. 2018 Aug;42(4):446-56.e1.

46 Pan H-Y, Guo L, Li Q. Changes of serum omentin-1 levels in normal subjects and in patients with impaired glucose regulation and with newly diagnosed and untreated type 2 diabetes. Diabetes Res Clin Pract. 2010 Apr; 88(1):29-33.

47 Gołąbek K, Ostrowska Z, Ziora K, Oświęcimska J, Świętochowska E, Marek B, et al. Association between omentin-1, bone metabolism markers, and cytokines of the RANKL/RANK/OPG system in girls with anorexia nervosa. Endokrynol Pol. 2015;66(6): 514-20.

48 Oświęcimska J, Suwała A, Świętochowska E, Ostrowska Z, Gorczyca P, Ziora-Jakutowicz $\mathrm{K}$, et al. Serum omentin levels in adolescent girls with anorexia nervosa and obesity. Physiol Res. 2015;64(5):701-9.

49 Shulman GI. Cellular mechanisms of insulin resistance. J Clin Invest. 2000 Jul;106(2):1716.

50 Korner J, Punyanitya M, Taveras C, McMahon DJ, Kim HJ, Inabnet W, et al. Sex differences in visceral adipose tissue post-bariatric surgery compared to matched non-surgical controls. Int J Body Compos Res. 2008;6(3): 93-9. 